\title{
RADIAL VELOCITIES AND METALLICITIES OF RED GIANT STARS IN THE OLD OPEN CLUSTER NGC 7762
}

\author{
Giovanni Carraro ${ }^{1,4,5}$, Eugene A. Semenko ${ }^{2}$, and Sandro Villanova ${ }^{3}$ \\ ${ }^{1}$ Dipartimento di Fisica e Astronomia Galileo Galilei, Vicolo Osservatorio 3, I-35122, Padova, Italy; giovanni.carraro@unipd.it \\ ${ }^{2}$ Special Astrophysical Observatory, Russian Academy of Sciences, Nizhny Arkhyz 369167, Russia \\ 3 Departamento de Astronomía, Universidad de Concepción, Casilla 160-C, Concepción, Chile \\ Received 2016 October 20; revised 2016 November 3; accepted 2016 November 8; published 2016 December 7
}

\begin{abstract}
We present and discuss radial velocity and the very first metallicity measurements for nine evolved stars in the poorly known old open cluster NGC 7762. We isolated eight radial velocity cluster members and one interloper. Radial velocities are in good agreement with previous studies. NGC 7762 turns out to be of solar metallicity within the uncertainties $([\mathrm{Fe} / \mathrm{H}]=0.04 \pm 0.12)$. For this metallicity, the cluster age is $2.5 \pm 0.2 \mathrm{Gyr}$, and falls in an age range where only a few old open clusters are known. With respect to previous studies, we find a larger distance, implying the cluster is located $900_{-50}^{+70} \mathrm{pc}$ from the Sun. For most of the elements we measure solar-scaled abundance ratios. We searched the literature for open clusters of similar age in the solar vicinity and found that NGC 7762 can be considered a twin of Ruprecht 147, a similar age cluster located only 300 pc from the Sun. In fact, as well as age, metallicity and abundance ratios are very close to Ruprecht 147 values within the observational uncertainties.
\end{abstract}

Key words: open clusters and associations: general - open clusters and associations: individual (NGC 7762)

\section{INTRODUCTION}

The last decade witnessed a significant accumulation of good quality, high-resolution, spectroscopic metallicity measurements for old open clusters in the Galactic disk. Metallicity, together with abundance ratios for a large sample of elements, of old open clusters of different age and location in the disk are the natural constraints for chemical evolution models that aim at understanding how the disk of the Milky Way formed and evolved. Essentially, this is because old open clusters span a wide range of ages, and are almost ubiquitous in the disk, thus allowing an observational age-metallicity relation, the actual radial abundance gradients, to be constructed and their evolution in time (Magrini et al. 2009). Additionally, open clusters offer the obvious advantage that the abundance of elements can be derived in statistical fashion, since no evidence has been found so far that they deviate from the single stellar population concept.

In this work, we present a spectroscopic investigation of the old open cluster NGC 7762, for which no abundance analysis has been performed to date.

The cluster was studied for the first time by Chincarini (1966), who in a short note provided photographic $U B V$ photometry of 18 stars, and preliminarily suggested a distance of $1 \mathrm{kpc}$ and a reddening $E(B-V)=1.02$. The same author recommended more data need to be obtained to extend the cluster main sequence (MS) for fainter magnitudes. Zakharova (1972) obtained an independent photographic data-set, of similar photometry depth, and derived a distance of $890 \mathrm{pc}$ and a reddening $E(B-V)=0.89$. She also provided an estimate of the mass $\left(\sim 400 M_{\odot}\right.$, and argued that the cluster is similar to the more famous NGC 752, NGC 1245, and NGC 7789, which implies an age above 1 Gyr. More recently, Patat \& Carraro (1995) acquired the very first CCD photometry, extending the MS down to $V \sim 19.0$. They noticed, however, that the MS disappears at $V \sim 16.5$, and interpreted this as evidence of low-

\footnotetext{
${ }_{5}^{4}$ ESO, Alonso de Cordoba 3107, Santiago de Chile, Chile.

5 Visiting Astronomer, Special Astrophysical Observatory.
}

mass star evaporation. In the same study they derived an age of $1.8 \mathrm{Gyr}$, a distance of $800 \mathrm{pc}$, and a reddening $E(B-V) \sim 0.85$. The most recent investigation is by Maciejewski et al. (2008), where the short distance derived previously is confirmed, but the derived age is larger (2.4 Gyr), and the reddening significantly smaller $(E(B-V)=0.59)$. Clearly, a significant improvement of the cluster's basic parameters can be obtained if metallicity is known.

We observed nine stars in the cluster red clump and derived their radial velocity and metallicity. The selected stars are shown in the color-magnitude diagrams (CMD) in Figure 1, and constructed using Maciejewski et al. (2008) photometric data. They are highlighted in red, and their properties are summarized in Table 1 .

The paper is organized as follows. In Section 2 we present observational material and the basic reduction strategy. Section 3 is devoted to radial velocities, while in Section 4 we discuss the derivation of the stars' atmospheric parameters. An abundance analysis is presented in Section 5. Section 6 is then dedicated to an update of the cluster fundamental parameters. In Section 7 we discuss our results and compare our abundance ratio determinations with the literature. Finally, Section 8 summarizes our findings.

\section{OBSERVATION AND DATA REDUCTION}

We observed NGC 7762 stars with the Main Stellar Spectrograph (MSS) ${ }^{6}$ of the $6 \mathrm{~m}$ telescope BTA at the Special Astrophysical Observatory in Nizhny Arkhyz, Russia. This instrument is installed in the Nasmyth 2 focus of the telescope. It is essentially a long-slit spectrograph, which in its standard mode is equipped with a differential circular polarization analyzer, and combined with a double image slicer designed by Chountonov (2004) and a rotating $1 / 4$ phase plate, to study mainly stellar magnetic fields. For the purpose of our observations, the instrument was set-up in its basic long-slit

\footnotetext{
6 www.sao.ru/hq/lizm/mss/en/index.html
} 


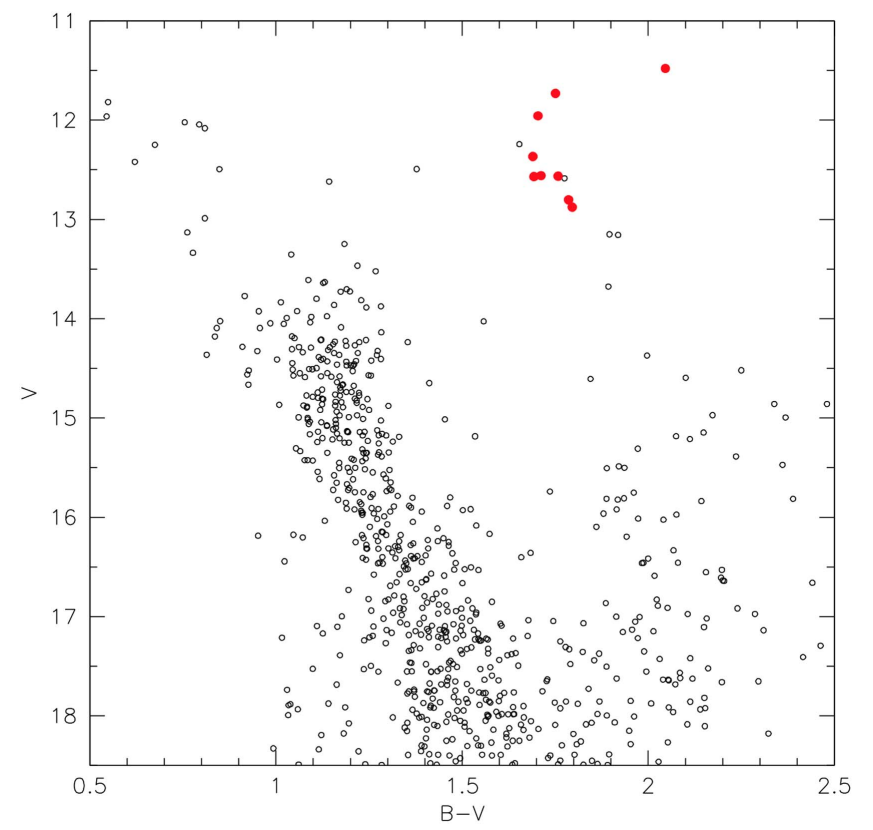

Figure 1. Color-magnitude diagram of NGC 7762. Red symbols indicate the stars for which we obtained spectroscopic data. Photometry is taken from Maciejewski et al. (2008).

mode. In this set-up, the MSS instrument covers the wavelength range $5940-6690 \AA$ with a resolution $\lambda / \Delta \lambda$ of $\sim 13,000$.

Observations were carried out on the night of 2016 August 12. The night was clear and stable, with typical seeing of $\sim 1$ arcsec. Besides the spectra of scientific targets (one exposure per target), we collected bias and dome flat frames (two sets of 10 images obtained at the beginning and at the end the night) and a set of ThAr spectra, for wavelength calibration purposes. Our intention was to observe all the stars in the magnitude range $11 \leqslant V \leqslant 13$, and redder than $(B-V) \sim 1.6$, but could obtain spectra only for nine stars in the allocated time.

Spectra were reduced in a standard way, which consists of the following steps: (1) construction of the master bias and subtraction of it from the scientific and calibration frames, (2) correction of data for stray light, (3) searching for the location of individual slices in two-dimensional (2D) images, (4) extraction of 1D spectra, (5) continuum normalization of the spectra, and (6) correction of wavelengths for the Earth's motion. All these procedures were carried out within ESOMIDAS package and its Zeeman extension. Spectra were normalized by the continuum level using the task continuum within the IRAF package. ${ }^{7}$ Wavelength solution was defined using the arc spectrum obtained closest in time to the scientific exposures.

An excerpt of the nine stars' spectra is shown in Figure 2.

\section{RADIAL VELOCITIES}

Radial velocities were measured by the fxcor package in IRAF, using a synthetic spectrum as a template calculated for a typical giant star with solar metallicity $\left(T_{\text {eff }}=4800 \mathrm{~K}, \log \right.$ $\left.(g)=2.50, v_{t}=1.50 \mathrm{~km} \mathrm{~s}^{-1}\right)$. All stars except for \#361 turned out to have the same velocity within a few $\mathrm{km} \mathrm{s}^{-1}$, and were

\footnotetext{
IRAF is distributed by the National Optical Astronomy Observatory, which is operated by the Association of Universities for Research in Astronomy, Inc., under cooperative agreement with the National Science Foundation.
}

considered members. The mean radial velocity for the cluster is $-46.5 \pm 0.8 \mathrm{~km} \mathrm{~s}^{-1}$. The typical error in radial velocity given by fxcor is $1.0 \mathrm{~km} \mathrm{~s}^{-1}$.

We compare in Table 2 our results with those of Casamiquela et al. (2016), who, in the context of the Open Cluster Chemical Abundances from Spanish Observatories (OCCASO) survey report radial velocity measurements for six stars in NGC 7762.

We have five stars in common with the OCCASO survey, and the comparison is extremely good (see Table 2).

\section{ATMOSPHERIC PARAMETERS}

Atmospheric parameters were obtained as follows. First, $T_{\text {eff }}$ was derived from the $B-V$ color using the relations by Alonso et al. (1999) and Ramirez \& Melendez (2005). Surface gravities $(\log (g))$ were obtained from the canonical equation

$$
\log \left(\frac{g}{g_{\odot}}\right)=\log \left(\frac{M}{M_{\odot}}\right)+4 \cdot \log \left(\frac{T_{\text {eff }}}{T_{\odot}}\right)-\log \left(\frac{L}{L_{\odot}}\right) .
$$

The bolometric correction was derived by adopting the relations from Alonso et al. (1999) and Flower (1996). We adopted initial values for reddening $E(B-V)$ and distance modulus $(m-M)_{V}$ from WEBDA and inferred stellar masses from isochrones (Bressan et al. 2012). We found $E(B-V)=0.71$ and $(m-M)_{V}=11.56$, and assumed a typical mass of $1.50 M_{\odot}$. Micro-turbulent velocity $\left(v_{\mathrm{t}}\right)$ was obtained from the relation of Gratton et al. (1996) that considers both temperature and gravity as parameters:

$$
v_{\mathrm{t}}=0.00119 \cdot T_{\mathrm{eff}}-0.90 \cdot \log (g)-2 .
$$

The input metallicity needed to obtain $T_{\text {eff }}$ from colours and for the isochrone fitting was assumed to be solar, which was later confirmed by the spectroscopic analysis (see below). The derived atmospheric parameters are listed in Table 3. The local thermodynamic equilibrium (LTE) program MOOG (Sneden 1973) was used for the abundance analysis coupled with atmosphere models by Kurucz (1970). We adopted the same line-list adopted in all our previous papers (Carraro et al. 2014a, 2014b).

\section{ELEMENTAL ABUNDANCES}

Si I, Ca I, Ti I, Fe I, Fe II, and Ni I abundances were estimated using the equivalent width (EQW) method. EQWs were obtained fitting a Gaussian to the spectral features. Na I and Ba II abundances were obtained using the spectrosynthesis method. For this purpose five synthetic spectra were generated for each line with a 0.25 dex abundance step and compared with the observed spectrum. The line-list and the methodology we used are the same used in previous papers (e.g., Villanova et al. 2013), so we refer to those articles for a detailed discussion of this point. Here we would like to stress that to measure $\mathrm{Ba}$ abundance hyperfine splitting was taken into account, as in our previous studies (e.g., Carraro et al. 2014a, 2014b). The derived elemental abundances are reported in Table 4.

Using Fe I and Fe II we were able to cross-check the atmospheric parameters obtained from the photometry in the following way. First of all for each star we calculated the mean $\mathrm{Fe}$ abundance from $\mathrm{Fe}$ I lines. Then we calculated for each $\mathrm{Fe}$ I and $\mathrm{Fe}$ II line the difference $\Delta(\mathrm{Fe})$ with respect to the mean 
Table 1

Basic Information of the Stars for which We Obtained Spectroscopic Data

\begin{tabular}{lccccccccc}
\hline \hline ID & R.A.(2000.0) & Decl.(2000.0) & $V$ & $B-V$ & $J$ & $H$ & $K$ & Exp (s) & $\frac{S}{N}$ \\
\hline 361 & $23: 48: 54.4$ & $68: 03: 52.1$ & 11.956 & 1.704 & 8.813 & 8.137 & 7.980 & 2400 \\
451 & $23: 49: 06.1$ & $67: 59: 08.6$ & 12.559 & 1.712 & 9.291 & 8.636 & 8.419 & 2700 & 80 \\
459 & $23: 49: 07.0$ & $67: 55: 24.2$ & 12.366 & 1.690 & 9.108 & 8.436 & 8.286 & 2700 \\
535 & $23: 49: 15.7$ & $68: 05: 32.1$ & 12.569 & 1.693 & 9.257 & 8.581 & 8.372 & 2700 & 70 \\
820 & $23: 49: 48.4$ & $68: 01: 35.1$ & 12.564 & 1.758 & 9.172 & 8.496 & 8.272 & 2700 & 75 \\
831 & $23: 49: 49.3$ & $68: 01: 07.3$ & 12.876 & 1.796 & 9.232 & 8.543 & 8.261 & 3600 \\
1195 & $23: 50: 31.4$ & $68: 01: 41.5$ & 11.730 & 1.751 & 8.431 & 7.781 & 7.518 & 3600 \\
1387 & $23: 50: 59.3$ & $68: 00: 36.6$ & 12.802 & 1.786 & 9.350 & 8.682 & 8.424 & 3600 \\
1455 & $23: 51: 12.1$ & $67: 56: 29.7$ & 11.479 & 2.046 & 7.594 & 6.719 & 6.469 & 110 \\
& & & & & & & & & 7000 \\
\hline
\end{tabular}

Note. IDs are taken from Maciejewski et al. (2008). The $\frac{S}{N}$ in the last column has been measured using a few $\AA$ continuum region at $6300 \AA$.

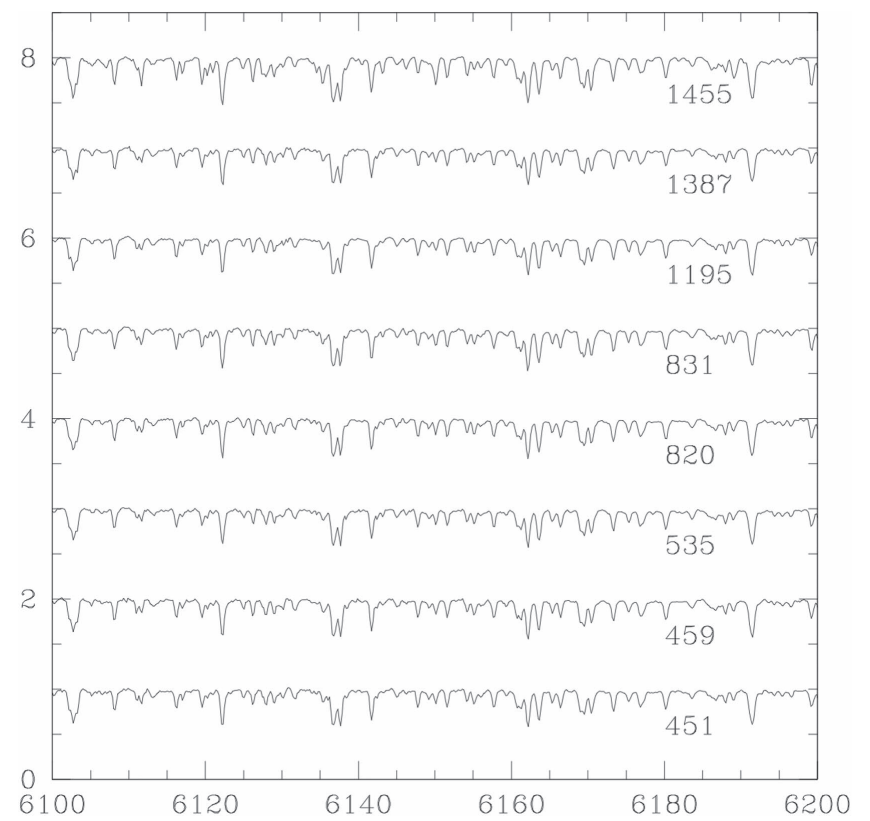

Figure 2. Excerpt of the nine individual spectra in the indicated wavelength range. Spectra are arbitrarily shifted in the vertical axis, for illustration purposes.

value. Finally we reported these differences in Figure 3 for all the stars. In the upper panel we plot $\Delta(\mathrm{Fe})$ as a function of the excitation potential (E.P.) and in the lower panel as a function of the reduced EW. ${ }^{8}$ We used black points for Fe I lines and red points for Fe II lines. In both panels Fe I lines were fitted with a straight blue line. The slope of each line is given in the upper part of each panel. Both slopes are compatible with a flat trend within $1 \sigma$. This means that the temperature and microturbulence scales we are using are reliable. In the same way we report in the upper panel the difference between the mean $\mathrm{Fe} \mathrm{I}$ and Fe II abundances. Also in this case the difference is compatible with zero within $1 \sigma$ and we conclude that the gravity scale we are using is reliable.

In order to estimate the uncertainties associated with these estimates, we considered star \#1387, and repeated the same process as above at varying $T_{\text {eff }}$ by $50^{\circ} \mathrm{K}, \log (g)$ by $0.20, v_{T}$ by $0.1 \mathrm{~km} \mathrm{~s}^{-1}$, and $[\mathrm{Fe} / \mathrm{H}]$ by 0.1 dex. Table 5 summarizes our results. The dependence on the limited spectra $\frac{S}{N}$ is also show.

\footnotetext{
8 The reduced equivalent width (R.E.W.) is defined as $\log _{10}\left(\frac{\text { EquivalentWidth }}{\lambda}\right)$.
}

Table 2

Radial Velocity Measurement for the Nine Program Stars in NGC 7762

\begin{tabular}{lcccc}
\hline \hline ID & R.A.(2000.0) & Decl.(2000.0) & $V_{R}$ & $V_{R, \text { OCCASO }}$ \\
\hline & & & $\left(\mathrm{km} \mathrm{s}^{-1}\right)$ & $\left(\mathrm{km} \mathrm{s}^{-1}\right)$ \\
361 & $23: 48: 54.4$ & $68: 03: 52.1$ & 36.4 & $\ldots$ \\
451 & $23: 49: 06.1$ & $67: 59: 08.6$ & -45.6 & $-45.9 \pm 0.7$ \\
459 & $23: 49: 07.0$ & $67: 55: 24.2$ & -48.8 & $\ldots$ \\
535 & $23: 49: 15.7$ & $68: 05: 32.1$ & -47.9 & $-45.5 \pm 0.6$ \\
820 & $23: 49: 48.4$ & $68: 01: 35.1$ & -46.2 & $-45.7 \pm 0.7$ \\
831 & $23: 49: 49.3$ & $68: 01: 07.3$ & -44.4 & $-47.0 \pm 0.8$ \\
1195 & $23: 50: 31.4$ & $68: 01: 41.5$ & -50.2 & $\ldots$ \\
1387 & $23: 50: 59.3$ & $68: 00: 36.6$ & -45.4 & $-45.6 \pm 0.8$ \\
1455 & $23: 51: 12.1$ & $67: 56: 29.7$ & -43.8 & $\ldots$ \\
\hline
\end{tabular}

Note. Literature values are from Casamiquela et al. (2016).

Table 3

Atmospheric Parameters for Member Stars

\begin{tabular}{lccc}
\hline \hline ID & $T_{\text {eff }}$ & $\log g$ & $v_{t}$ \\
\hline & $\left({ }^{\circ} \mathrm{K}\right)$ & & $\left(\mathrm{km} \mathrm{s}^{-1}\right)$ \\
\hline 451 & 4800 & 2.655 & 1.323 \\
459 & 4845 & 2.601 & 1.424 \\
535 & 4839 & 2.679 & 1.347 \\
820 & 4713 & 2.609 & 1.260 \\
831 & 4640 & 2.692 & 1.098 \\
1195 & 4726 & 2.283 & 1.569 \\
1387 & 4659 & 2.674 & 1.137 \\
1455 & 4214 & 1.833 & 1.365 \\
\hline
\end{tabular}

The column indicated with Tot is the quadratic sum of the various contributions, while the term $\mathrm{Obs}$ indicates the dispersion of the ratio measurements among the eight member stars.

\section{CLUSTER BASIC PARAMETERS REVISITED}

The abundance analysis carried out in previous sections yields a mean metal abundance $[\mathrm{Fe} / \mathrm{H}]=0.04 \pm 0.12$, compatible with the solar value. From the individual values (see Table 4) one can notice some spread, which, however, is totally compatible with the observational errors. Using this new, spectroscopic, metal abundance estimate, we can now derive NGC 7762 fundamental parameters, and for this purpose we employ the Padova suite of stellar models and isochrones (Bressan et al. 2012). Our results are summarized in Figure 4. We favor an age of $2.5 \pm 0.2 \mathrm{Gyr}$. The corresponding isochrones nicely fit both the cluster MS 
Table 4

Abundance Analysis for Member Stars

\begin{tabular}{lrcrrrr}
\hline \hline ID & {$[\mathrm{Fe} / \mathrm{H}]$} & {$[\mathrm{Si} / \mathrm{Fe}]$} & {$[\mathrm{Ca} / \mathrm{Fe}]$} & {$[\mathrm{Ti} / \mathrm{Fe}]$} & {$[\mathrm{Ni} / \mathrm{Fe}]$} & {$[\mathrm{Na} / \mathrm{Fe}]$} \\
\hline 451 & 0.08 & 0.08 & 0.18 & 0.00 & 0.17 & -0.26 \\
459 & 0.09 & 0.25 & 0.26 & 9.99 & 0.17 & -0.14 \\
535 & -0.02 & 9.99 & 0.06 & 0.10 & 0.11 & -0.19 \\
820 & 0.09 & 0.34 & 0.05 & -0.02 & 0.15 & -0.29 \\
831 & 0.18 & 0.23 & 0.04 & 9.99 & 0.29 & -0.28 \\
1195 & -0.15 & 0.29 & 0.11 & 0.02 & 0.99 & 0.38 \\
1387 & -0.12 & 0.34 & -0.10 & -0.06 & 0.20 & 0.47 \\
1455 & 0.15 & 9.99 & 9.99 & 0.26 & 0.09 & -0.06 \\
\hline
\end{tabular}
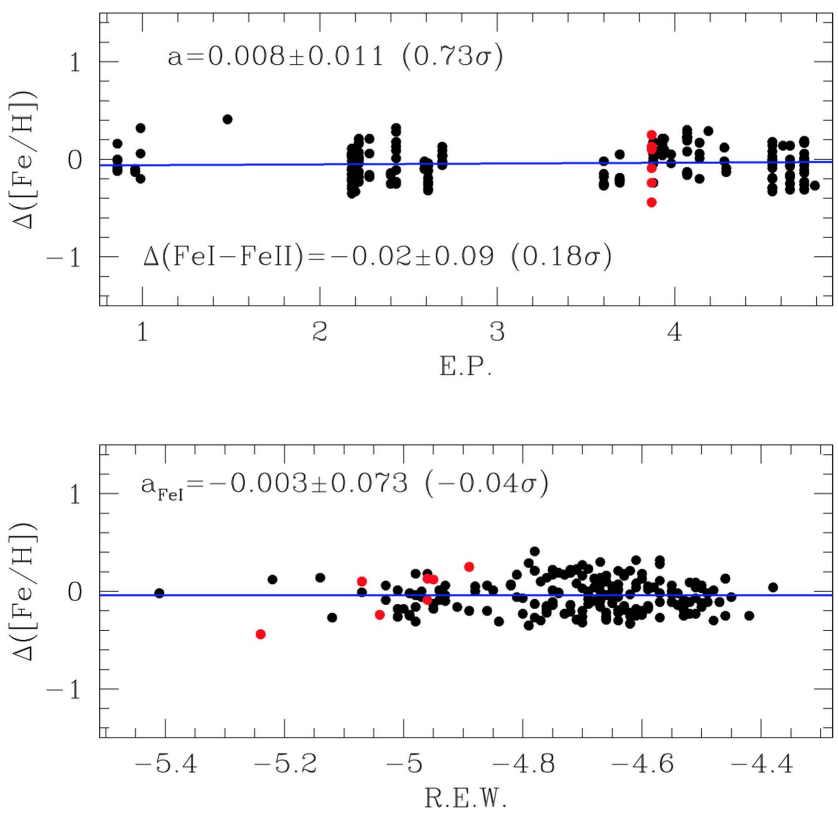

Figure 3. Difference of $[\mathrm{Fe} / \mathrm{H}]$ measurements from $\mathrm{Fe} \mathrm{I}$ and $\mathrm{Fe}$ II with respect to the mean $\mathrm{Fe}$ abundance as a function of the excitation potential (E.P.) and the reduced equivalent width (R.E.W.). See text for details.

Table 5

Error Budget

\begin{tabular}{lccccccc}
\hline \hline Ratio & $T_{\text {eff }}$ & $\log (g)$ & $v_{t}$ & {$[\mathrm{Fe} / \mathrm{H}]$} & $\frac{S}{N}$ & Tot & Obs \\
\hline$[\mathrm{Si} / \mathrm{Fe}]$ & 0.04 & 0.03 & 0.03 & 0.03 & 0.05 & 0.08 & 0.10 \\
{$[\mathrm{Ca} / \mathrm{Fe}]$} & 0.05 & 0.02 & 0.05 & 0.02 & 0.07 & 0.10 & 0.11 \\
{$[\mathrm{Ti} / \mathrm{Fe}]$} & 0.05 & 0.01 & 0.02 & 0.01 & 0.08 & 0.10 & 0.12 \\
{$[\mathrm{Fe} / \mathrm{H}]$} & 0.06 & 0.02 & 0.07 & 0.02 & 0.03 & 0.10 & 0.12 \\
{$[\mathrm{Ni} / \mathrm{Fe}]$} & 0.02 & 0.02 & 0.01 & 0.01 & 0.07 & 0.08 & 0.07 \\
{$[\mathrm{Na} / \mathrm{Fe}]$} & 0.02 & 0.03 & 0.05 & 0.01 & 0.08 & 0.10 & 0.13 \\
{$[\mathrm{Ba} / \mathrm{Fe}]$} & 0.02 & 0.06 & 0.05 & 0.02 & 0.03 & 0.09 & 0.08
\end{tabular}

Note. Each column reports the error dependence on the various atmospheric parameters.

and the red giant clump. This solution implies a reddening $E(B-V)=0.62 \pm 0.03$ and an apparent distance modulus $(m-M)_{V}=11.7 \pm 0.1$. Uncertainties are estimated by visual inspection, shifting the isochrone in the horizontal and vertical direction until the fit becomes unacceptable.

While our reddening value is compatible with the Maciejewski et al. (2008) estimate, we find some disagreement ( $\approx 10 \%$ larger) for the distance modulus estimate, despite the fact we are using their photometry and roughly the same age.

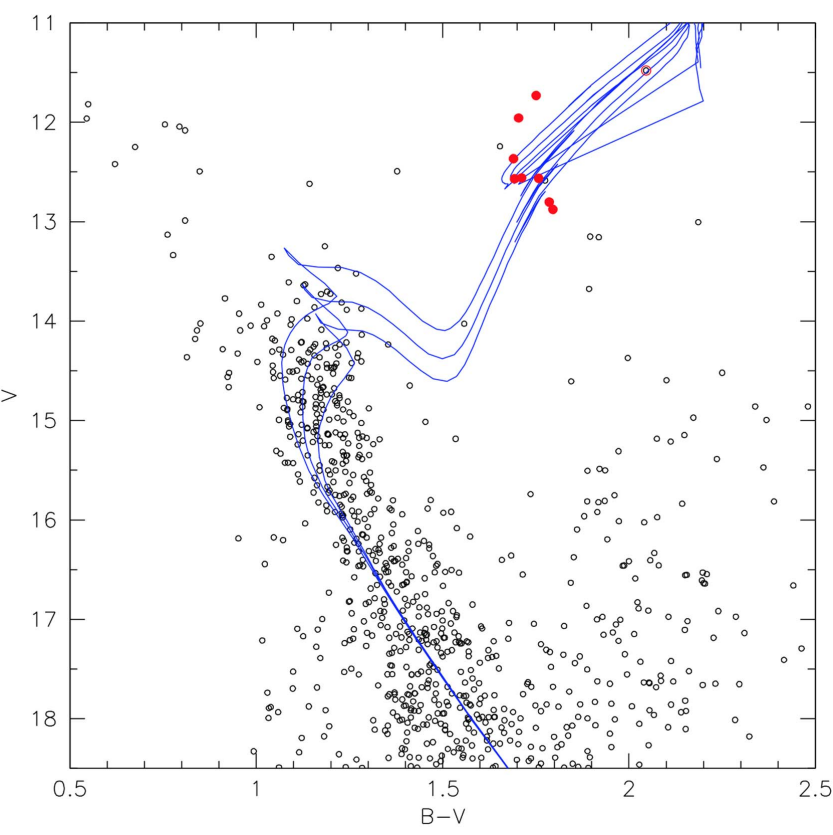

Figure 4. NGC 7762 basic parameters' solution. Isochrones in blue are shown for ages of 2.0, 2.5, and $3.0 \mathrm{Gyr}$, and superimposed for $E(B-V)=0.62$ and $(m-M)=11.7$. Red filled symbols indicate the member stars for which spectroscopy has been obtained in this study. The empty symbol refers to the non-member.

However, if one considers carefully their isochrone solution (their Figure 3), it is immediately clear that the fit is poor both in optical and in infrared. In fact, the optical fit completely misses the clump, while the infrared fit matches the clump position, but not the cluster TO.

The distribution of the observed stars in the CMD deserves more attention. We have adopted as the position of the red giant clump the location of the three stars \#451, \#535, and $\# 820$, that have the same magnitude level. According to this solution, we would argue that the two fainter and slightly redder stars \#831 and \#1387 are red giant branch (RGB) stars, while the remaining stars are most probably asymptotic giant branch (AGB) stars, which are expected to be present in a cluster of this age. The isochrone mismatch in color is not particularly worrisome, given the various uncertainties affecting models in these evolutionary phases: mixing length parameter calibration, transformation from theoretical to observational plane, atmosphere models, and so forth.

Our new distance estimate then places NGC 7762 at $900_{-50}^{+70} \mathrm{pc}$ from the Sun.

This updated set of parameters (reddening and apparent distance modulus) slightly differs from the one we adopted from WEBDA as input to estimate stars' atmospheric 

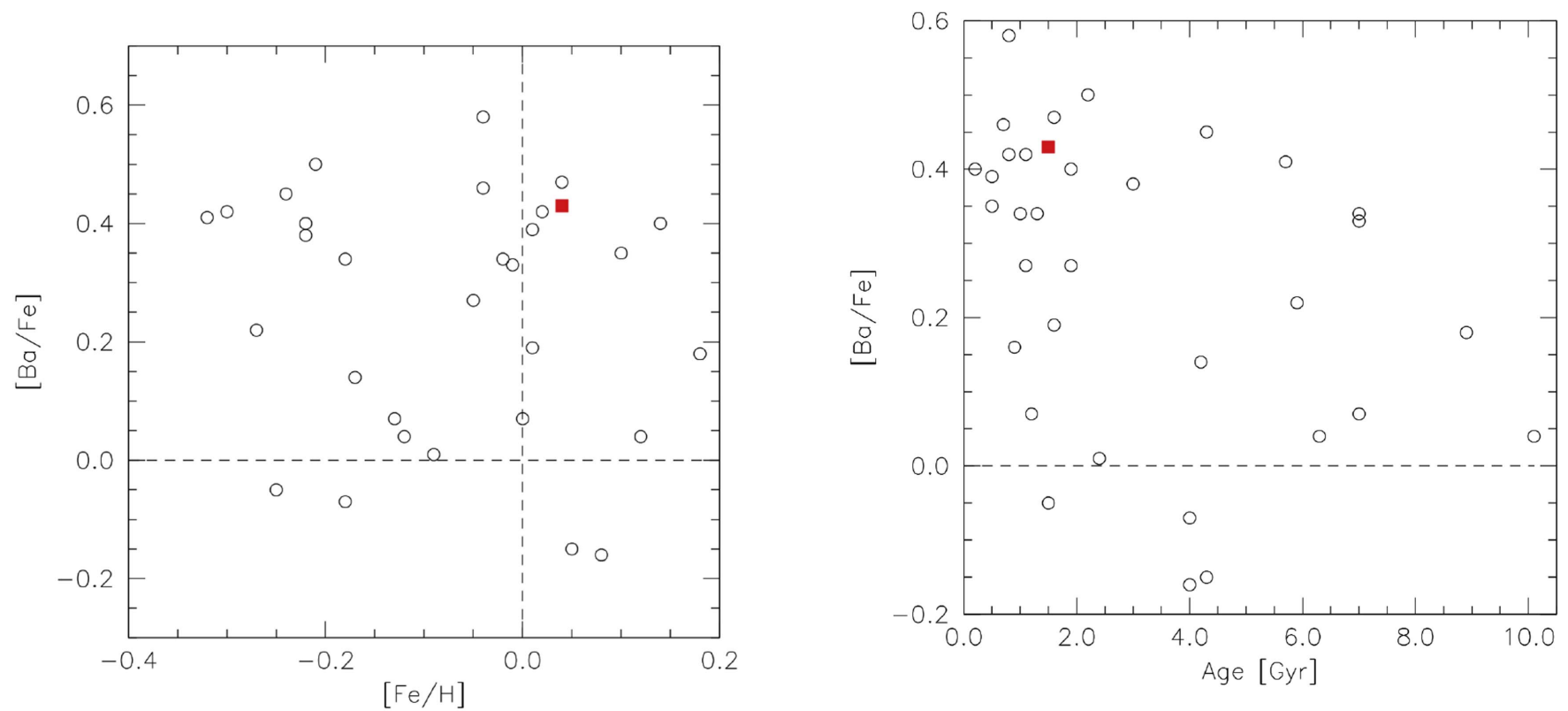

Figure 5. Trend of $[\mathrm{Ba} / \mathrm{Fe}]$ abundance ratio vs. metallicity (left panel) and vs. age (right panel) for a compilation of old open clusters. The red square indicates NGC 7762 .

parameters. We investigated this issue in details and found that there is no implication for $\log (g)$, since a difference in apparent distance modulus of a few hundredths magnitudes only (within the errors) would produce a difference in $\log (g)$ of 0.07 . Reddening is on the other hand critical for the derivation of $\log \left(T_{\text {eff }}\right)$. In our case, if we take errors into consideration the difference in $E(B-V)$ is $0.06 \mathrm{mag}$ ( $1 \sigma$ difference), which causes a difference in $\log \left(T_{\text {eff }}\right)$ of $80^{\circ}$. Stars would then be slightly cooler, and their metallicity slightly lower (by $0.05 \mathrm{dex}$ ), which on the other hand is largely compatible with our estimate.

\section{DISCUSSION}

Alongside metal abundance, we also derived a few abundance ratios, as listed in Table 4. Their uncertainties are listed in Table 5. We are going to compare our findings with the population of Galactic old open clusters for which such ratios are available, to put NGC 7762 into context.

\subsection{Metallicity and $\alpha$ Elements}

With an age of $2.5 \mathrm{Gyr}$ and a metallicity of $[\mathrm{Fe} / \mathrm{H}]=$ $0.04 \pm 012$, NGC 7762 looks like a typical old open cluster in the solar neighborhood. There are not many old open clusters closer than $\sim 1 \mathrm{kpc}$ to the Sun to compare our findings with. If we generate such a list from WEBDA ${ }^{9}$ we find only one cluster sharing the same age as NGC 7762: Ruprecht 147 (Curtis et al. 2013). According to these authors, Ruprecht 147 is also a $2.5 \pm 0.25 \mathrm{Gyr}$ old open cluster. Both clusters, by the way, are quite spread over the sky, this being a combined effect of their vicinity to the Sun and the advanced stage of dynamical evolution. Interestingly, Ruprecht 147 has a metallicity of $[\mathrm{Fe} / \mathrm{H}]=0.07 \pm 0.03$, again virtually identical to NGC 7762 .

We have two $\alpha$ element abundance ratios (for $\mathrm{Si}$ and $\mathrm{Ti}$ ) in common with Curtis et al. (2013) and Pakhomov et al. (2009). Our mean $[\mathrm{Ti} / \mathrm{Fe}]=0.08$ is compatible with their

\footnotetext{
9 http://www.univie.ac.at/webda/clusterselall.html
}

$[\mathrm{Ti} / \mathrm{Fe}]=0.03$ within the uncertainties, while our $[\mathrm{Si} / \mathrm{Fe}]=$ 0.25 is clearly overabundant with respect to the Sun compare with their $[\mathrm{Si} / \mathrm{Fe}]=0.02$. We remark however that the involved number of stars is relatively low and both studies might be affected by low number statistics. Overall the two clusters appear to share common properties, but NGC 772 is more distant from the Sun $(\sim 900 \mathrm{pc})$ than Ruprecht 147 $(\sim 300 \mathrm{pc})$.

\subsection{Iron Peak Elements}

Our wavelength coverage allows us to measure only one iron-peak element: Ni. Our mean value for $[\mathrm{Ni} / \mathrm{Fe}]$ is $0.14 \pm 0.08$, while Curtis et al. (2013) found for Ruprecht 417 a value of $[\mathrm{Ni} / \mathrm{Fe}]=-0.02 \pm 0.05$. Given the low number statistics, we conclude that this element is close to solar.

\subsection{Neutron-capture Elements}

Mishenina et al. (2015, and references therein) showed that Galactic old open clusters for which Ba was measured exhibit a clear $\mathrm{Ba}$ overabundance with respect to the Sun. The overabundance correlates with age, since it increases with decreasing age. In addition, for clusters younger than 3-4 Gyr, the $\mathrm{Ba}$ abundance shows a large scatter. In Figure 5 we compare the NGC $7762[\mathrm{Ba} / \mathrm{Fe}]$ ratio with a compilation of old open clusters from Mishenina et al. (2013, 2015) and Jacobson \& Friel (2013). Although incomplete, this compilation has the advantage that the typical age and metallicity ranges for Galactic old open clusters are well covered. The comparison for NGC 7762 is shown in the two panels of Figure 5, where our cluster is identified with a red square. The left panel shows the well-known large scatter of $[\mathrm{Ba} / \mathrm{Fe}]$ at any metallicity, and NGC 7762 comfortably sits among the other old open clusters. In the right panel, we show that the addition of NGC 7772 does not change the general scenario. The mean $[\mathrm{Ba} / \mathrm{Fe}]$ overabundance we measured from our eight giant stars $([\mathrm{Ba} / \mathrm{Fe}]=0.43 \pm 0.15)$ follows the general trend. 


\section{CONCLUSION}

In this work we have presented the very first abundance analysis for the Galactic old open cluster NGC 7762. This cluster was in fact poorly known, mostly because of its large extent and the severe field star contamination. We observed nine stars in the evolved region of the CMD, and isolated eight members: three clump stars, two RGB stars, and three possible AGB stars. Our results can be summarized as follows:

1. Our spectroscopic analysis reveals that the cluster is of solar metal content $([\mathrm{Fe} / \mathrm{H}]=0.04 \pm 0.12)$, which we would expect from its location close to the Sun.

2. Our best fit age is $2.5 \pm 0.5 \mathrm{Gyr}$, which is mostly in line with previous - purely photometric — estimates.

3. With respect to previous investigations we argue in this study that the distance is slightly larger, and position the cluster $900 \mathrm{pc}$ from the Sun. We confirm previous reddening estimates within the observational uncertainties.

4. Beside metallicity, we found that the abundance ratios we could measure also reveal a solar-scaled mixture of elements in the cluster.

5. In NGC 7762 barium is over-abundant with respect to the Sun. This result follows the trend of the general population of old open clusters in the Milky Way.

Overall, the fundamental parameters we obtained in this study make NGC 7762 a twin of Ruprecht 147, a coeval cluster located only $300 \mathrm{pc}$ from the Sun.

G. Carraro thanks the CAO staff, in particular Gennady Valyavin, for kind hospitality, and G. Galazutdinov for his help during the preparation of this project. The visit to CAO has been financially supported by the ESO Office for Science in
Chile. S. Villanova gratefully acknowledges support from the Chilean BASAL and Centro de Excelencia en Astrofsica y Tecnologas Afines (CATA) grant PFB-06/2007. We made extensive use of the WEBDA databased, maintained by $\mathrm{E}$. Paunzen at Masaryk University in Brno.

Facility: CAO.

Software: MIDAS, IRAF.

\section{REFERENCES}

Alonso, A., Arribas, S., \& Martínez-Roger, C. 1999, A\&AS, 140, 261

Bressan, A., Marigo, P., Girardi, L., et al. 2012, MNRAS, 427, 127

Carraro, G., Monaco, L., \& Villanova, S. 2014a, A\&A, 568, 86

Carraro, G., Villanova, S., Monaco, L., et al. 2014b, A\&A, 562, 39

Casamiquela, L., Carrera, R., Jordi, C., et al. 2016, MNRAS, 548, 3150

Chincarini, G. 1966, MmSAI, 37, 423

Chountonov, G. A. 2004, in Proc. Int. Conf., Magnetic Stars, ed. Yu. Glagolevskij, D. Kudryavtsev, \& I. Romanyuk, 286

Curtis, J. L., Wolfgang, A., Wright, J. T., Brewer, J. M., \& Johnson, J. A. 2013, AJ, 145,134

Flower, P. J. 1996, ApJ, 469, 355

Gratton, R. G., Carretta, E., \& Castelli, F. 1996, A\&A, 314, 191

Jacobson, H. R., \& Friel, E. D. 2013, AJ, 145, 107

Kurucz, R. L. 1970, SAOSpecial Report No. 309

Maciejewski, G., Boeva, S., Georgiev, Ts., et al. 2008, BaltA, 17, 51

Magrini, L., Sestito, P., Randich, S., \& Galli, D. 2009, A\&A, 494, 95

Mishenina, T., Korotin, S., Carraro, G., Kovtyukh, V., \& Egorova, I. 2013, MNRAS, 433, 1436

Mishenina, T., Pignatari, M., Carraro, G., et al. 2015, MNRAS, 446, 3651

Pakhomov, Y. V., Antipova, L. I., Boyarchik, A. A., Zhao, G., \& Liang, Ya. 2009, ARep, 53, 660

Patat, F., \& Carraro, G. 1995, A\&AS, 114, 281

Ramirez, I., \& Melendez, J. 2005, ApJ, 626, 465

Sneden, C. 1973, ApJ, 184, 839

Villanova, S., Geisler, D., Carraro, G., Moni Bidin, C., \& Munoz, C. 2013, ApJ, 778, 186

Zakharova, P. E. 1972, ATsir, 740, 6 